\title{
Diabetes Mellitus and the Risk for Developing Cancer
}

Shane Malachy Markey, Marie A Congiusta

\begin{abstract}
Diabetes mellitus is a serious and growing health care problem, globally.

The 'westernization' of diets around the world has led to an increase in obesity. O ther contributing factors such as smoking, lack of physical activity and aging has led to epidemiological concerns regarding the link between diabetes and cancer. Research evidence suggests that patients with diabetes may be at greater risk for the development of some forms of cancer. The patient being discussed in this case report presented to New York University College of Dentistry (NYUCD), with a history that included type 2 diabetes mellitus, seeking comprehensive dental care. During the interim between his initial exam and following appointment, a lesion had developed on the palate, which was subsequently diagnosed as adenocarcinoma. This prompted the review of current literature, regarding the link between diabetes and the development of various cancers.
\end{abstract}

Keywords: Diabetes, Diabetes mellitus, Glycemic control, Cancer, Oral cancer, Adenocarcinoma, Smoking, Obesity.

How to cite this article: Markey SM, Congiusta MA. Diabetes Mellitus and the Risk for Developing Cancer. Int J Experiment Dent Sci 2013;2(1):41-43.

\section{Source of support $\mathrm{Nil}$}

Conflict of interest: None declared

\section{INTRODUCTION}

Diabetes mellitus is a well-recognized, serious worldwide health problem. As experts of the oral cavity, we play a vital role in the health promotion of our patients. It is often the dentist who first suspects and initiates the process of diagnosing underlying medical conditions.

Research and epidemiological evidence suggests that patients who have diabetes are at greater risk for developing various forms of cancers. Smoking, obesity, sedentary lifestyles, age and race are also contributing risk factors. ${ }^{1,2}$

As health care professionals we see many patients in our day-to-day practice. We have an obligation to inform and educate them whenever possible. By creating awareness we can hel p patients avoid missed diagnosis as well as delays in proper care. Understanding the link between diseases like diabetes and cancer can help save lives.

This case report describes the management of a patient with a 20-year history of diabetes. U pon identification and diagnosis of adenocarcinoma of the soft palate, a review of the literature was done to identify any associations that may exist between diabetes and cancer.

\section{CASE REPORT}

A 55-year-old Hispanic male presents to NY U CD in need of treatment for his periodontal condition. $\mathrm{He}$ is a type 2 diabetic, well controlled by oral hypoglycemic medications. He is a smoker of 37 plus years, with a pack a day history. He had attempted to quit several times in the past but was unsuccessful. Smoking cessation assistance was offered, however, due to current personal hardships he was unable to commit to any of our recommendations. A past history of occasional drinking was reported. The patient was 5 foot 6 inches tall and weighed $125 \mathrm{~kg}$. Respiration rate was 16 with a pulse rate of 72 . $\mathrm{H}$ is blood pressure readings at initial exam were 128/85 and 131/79, controlled by antihypertensives. Patient also reported a history of myocardial infarction. With a medical history that includes cardiovascular disease, in addition to smoking, this patient was at high risk for further worsening of his diabetic condition as well as at high risk for cancer. ${ }^{3,4} \mathrm{~A}$ lso, recent studies show that diabetes and periodontal disease may have reciprocal deleterious effects. ${ }^{4}$

A t patients initial visit, a full mouth series of radiographs were taken (Fig. 1) as well as alginate impressions for diagnostic study models. A thorough extraoral/intraoral, soft and hard tissue examination was completed. Findings included generalized moderate to severe localized periodontitis. M obility of grade II or more was identified in several teeth. The patient had a palatally impacted cuspid in the upper left anterior quadrant, which he was aw are of.

A fter a 5-week lapse the patient returned for completion of treatment planning and initiation of care. U pon reexamination the patient had developed a unilateral swelling in the upper right palate that measured $15 \times 15 \mathrm{~mm}$. A sizable lesion, not initially present, the patient had been unaware of its development (Figs $2 A$ and $B$ ). The patient was immediately informed and referred to the oral medicine department of NY UCD for further evaluation. Prior to biopsy a presumptive clinical diagnosis of pleomorphic adenoma was considered. An incisional biopsy was performed and sent for histopathological study. The laboratory reported a finding of adenocarcinoma of minor salivary gland origin. Surgical removal was performed to remove the malignancy ( $F$ igs $3 \mathrm{~A}$ and $\mathrm{B}$ ). 


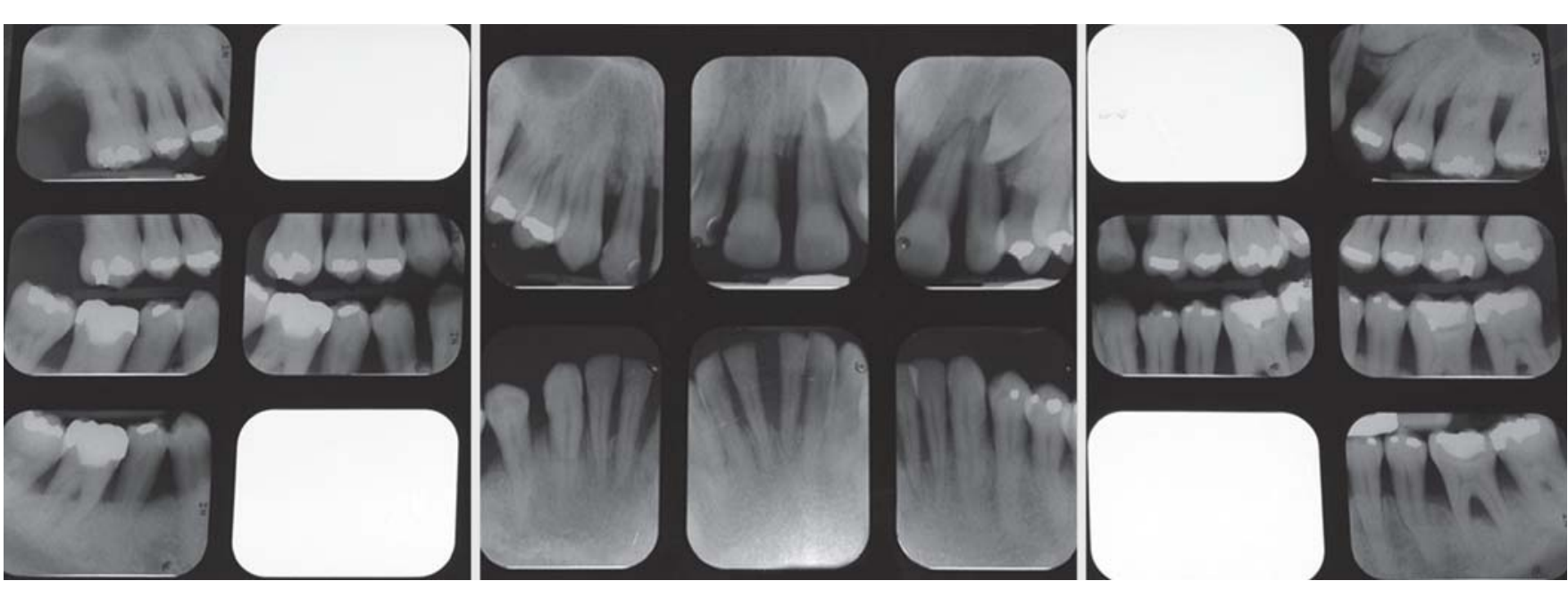

Fig. 1: Preoperative full mouth series radiographs
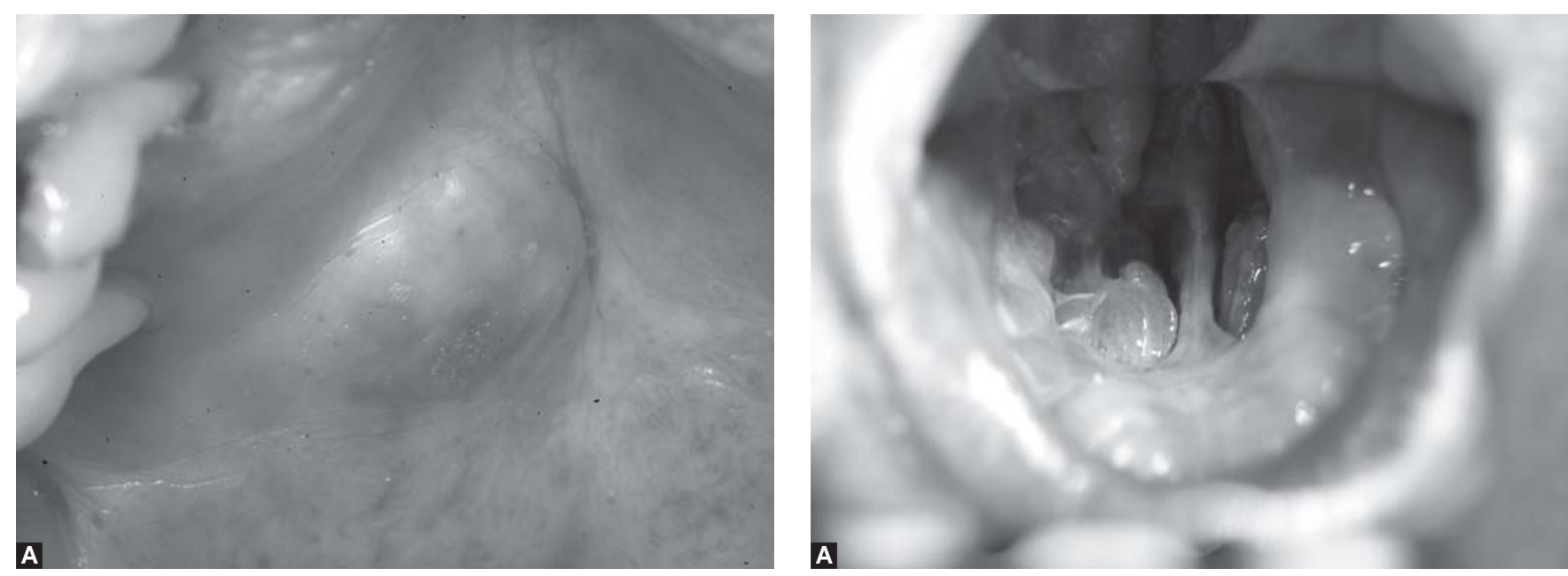

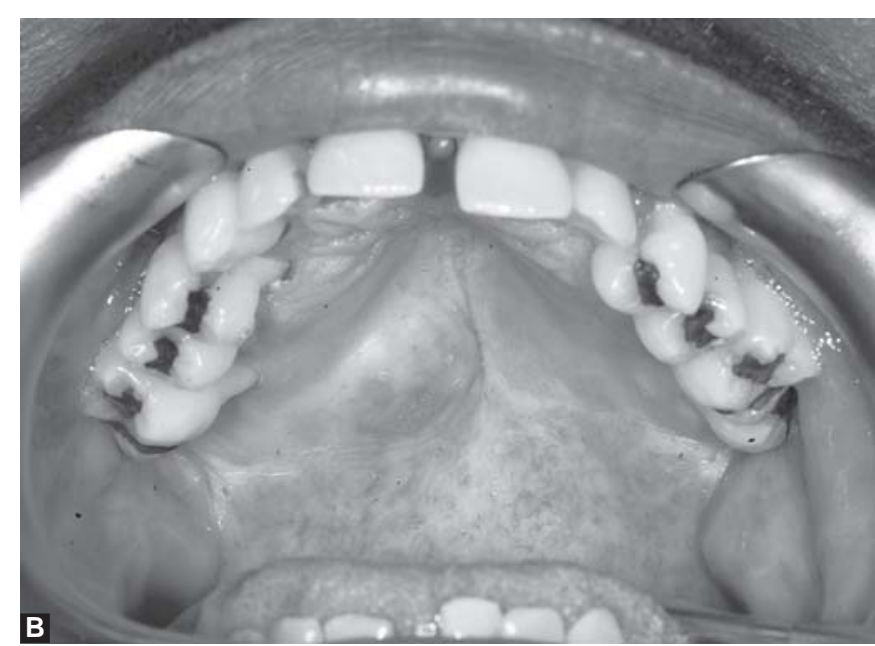

Figs 2A and B: Intraoral preoperative images showing palatal lesion

The initial treatment plan was revised after diagnosis of malignancy to extraction of all upper teeth and a full maxillary denture with obturator designed to occlude oral antral communication left from surgical excision (Figs 4A and $B$ ). The lower arch required extraction of periodontally hopeless teeth and fabrication of a cast partial denture.

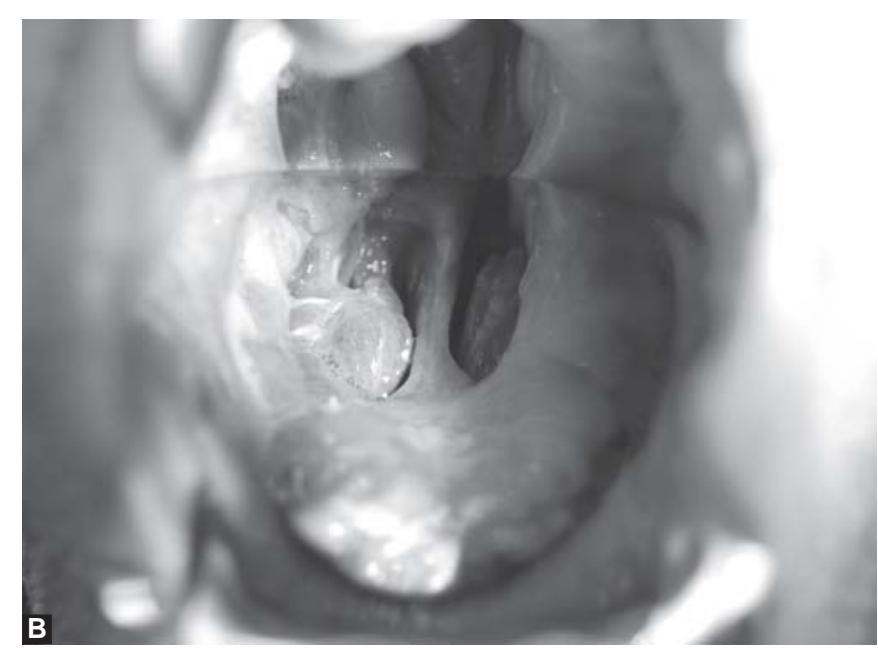

Figs $3 \mathrm{~A}$ and $\mathrm{B}$ : Intraoral postoperative images

\section{DISCUSSION}

Current evidence suggests a strong association between diabetes mellitus and the development of liver, pancreatic, endometrial, breast, colon/rectum and bladder cancer. ${ }^{2}$ It has been shown that diabetes has been linked to a decreased incidence of prostate cancer, however, for some cancer sites 

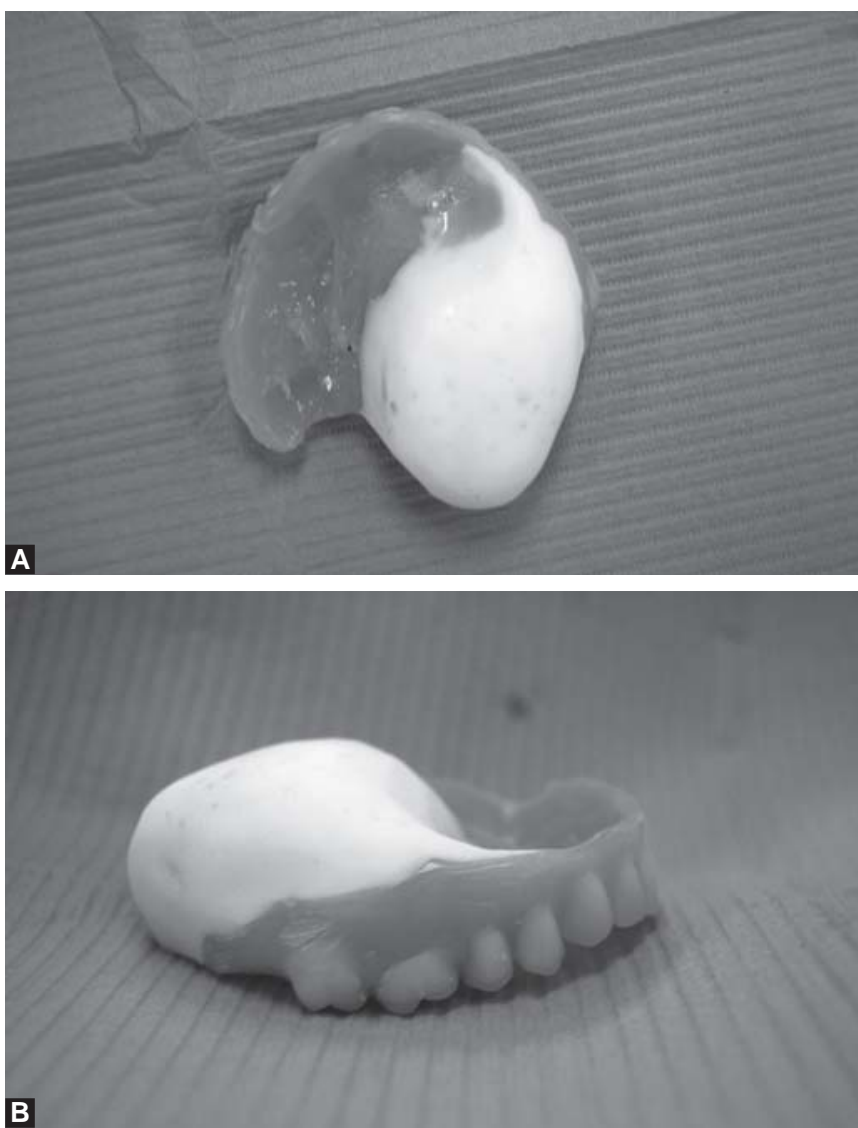

Figs 4A and B: Complete upper denture with silicone obturator

there appears to be no association or the evidence is inconclusive. ${ }^{1}$

The mechanisms proposed for the link between diabetes and cancer, include: Hyperinsulinemia, hyperglycemia and chronic inflammation. ${ }^{1}$

Chronic inflammation may result in an increase in the release of proinflammatory cytokines, which in turn can mediate changes in the neoplastic cell and al ter the response of a malignancy to diet, e.g. leading to increased tumor growth. ${ }^{1}$

The role of the insulin receptor has been studied and it has been shown that neoplastic cells overexpress this receptor and this may in turn mediate neoplastic growth and tumor differentiation. ${ }^{1,2}$

Some researchers suggest that a link exists between diabetic medications and cancer development. For example, some suggest that metformin is associated with a lower risk for cancer development as compared to exogenous insulin (Insulin Glargine/L antus), which has been associated with an increased risk for cancer. ${ }^{2,5}$ Others, however, suggest that the evidence is limited and any observed associations may be influenced by the progressive nature of the disease as well. ${ }^{1}$

Patient education should involve an understanding of current research, with an emphasis on prevention. Glycemic control, through diet, exercise and smoking cessation should be reinforced whenever possible. Early detection is of utmost importance and the dentist, as part of a patients' health management team, is obligated to inform patients when indicated. ${ }^{1,2}$

Patients with diabetes should be strongly encouraged to undergo appropriate and timely cancer screenings by recommended guidelines.

\section{SUMMARY}

In the case report described, diabetes is a possible influencing risk. However, due to other risk factors, especially the smoking history, one cannot identify the specific cause of the presenting adenocarcinoma. It is well know $n$ that smoking plays a major role in the initiation and progression of oral cancers, however the role of the insulin receptor, and its overexpression in diabetics may have also influenced the aggressive and acute nature of this tumor and its rapid growth pattern., ${ }^{1,6,7}$ The literature suggests that the incidence of cancer in diabetic patients is greater than in nondiabetic patients and further research in this area is needed.

\section{REFERENCES}

1. Giovannucci E, Harlan D, A rcher M, Bergenstal R, Gapstur S, Habel L, et al. Diabetes and Cancer: A Consensus Report. Diabetes Care 2010;33:1674-85.

2. Sunghwan S, K wang-W on K. Diabetes and Cancer: Is diabetes causally related to cancer? Diabetes M etab J 2011;35:193-98.

3. Ship J. Diabetes and Oral Health: An Overview. J Am Dent A ssoc 2003;134 Spec No:4S-10S.

4. Herman W W, K onzelman J L J r, Prisant L M , et al. N ew national guidelines on hypertension: A summary for dentistry. J A m Dent Assoc 2004;135:576-84.

5. B owker SL, M ajumdar SR, V eugelers P, J ohnson J A . I ncreased cancer-related mortality for patients with type 2 diabetes who use sulphonylureas or insulin. Diabetes Care 2006;29:254-58.

6. Haire-J oshu D, Glasgow RE, Tibbs TL. Smoking and diabetes. Diabetes Care 1999; 22:1887-98.

7. F oy CG, B ell RA, Farmer DF, G off DC J r, W agenknecht LE. Smoking and incidence of diabetes among us adults: Findings from the insul in resistance atherosclerosis study. Diabetes $C$ are 2005;28:2501-07.

\section{ABOUT THE AUTHORS}

\section{Shane Malachy Markey (Corresponding Author)}

4th Year DDS Candidate, Department of Cariology and Comprehensive Care, College of Dentistry, New Y ork University New Y ork, USA, e-mail: shanemarkey@ mac.com

\section{Marie A Congiusta}

Full Time Clinical Faculty and Instructor, Department of Cariology and Comprehensive Care, College of D entistry, N ew Y ork U niversity New Y ork, USA 